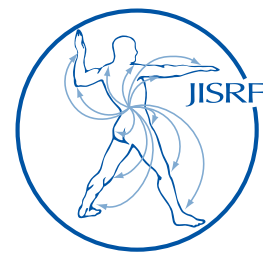

\title{
Combination of Accelerometer-Based Navigation and Force Sensor for Precise Bone Resection and Appropriate Soft Tissue Balancing in Total Knee Arthroplasty
}

\author{
Oshima $Y^{1}$, Fetto $J^{1}$
}

\section{Abstract}

Background: Precise bone resection and appropriate soft tissue balancing are considered indispensable in total knee arthroplasty (TKA). However, in most TKAs, only the experienced-based subjective physical "feel" of the surgeon, or either a computer-based navigation system or a soft tissue balancing system are applied to improve the results. In the present study, a combination of both an accelerometerbased navigation system and an electronic knee balancing force sensor were applied to attempt to obtain optimal outcomes.

Materials and Methods: An accelerometer-based navigation system and an electronic knee balancing force sensor were applied in combination in 5 TKAs. Thereafter, the incidence of radiographical outliers of the lower-extremity mechanical axis and the alignments of femoral and tibial components, and the incidence of intraoperative lateral retinacular release were evaluated and compared against those of 5 TKAs performed with the force sensor alone as a control.

Results: The posterior slope of the tibia was significantly improved in the TKAs performed with the combination of both devices $(\mathrm{P}=0.004)$. No lateral release was performed in any TKAs of either group.

Conclusion: TKAs performed under the combination of an accelerometer-based navigation system and an electronic knee balancing force sensor can obtain greater the accuracy of bone resection and appropriate soft tissue balancing.

Keywords: total knee arthroplasty (TKA); computer-assisted surgery (CAS); accelerometer-based navigation; electronic knee balancing system; bone resection; soft tissue balancing

Level of Evidence: AAOS Therapeutic Level IV

1 Yasushi Oshima, MD, PhD; Joseph Fetto, MD Division of Adult Reconstructive Surgery, Department of Orthopaedic Surgery, New York University Hospital for Joint Diseases, 1040 First Avenue, \#345, New York, NY 10022 USA

(Direct reprint requests to Yasushi Oshima)
(C) 2016 Oshima, Fetto. All rights reserved.

Reconstructive Review is a peer-reviewed, open-access orthopaedic journal devoted to publishing papers in the area of reconstructive arthroplasty. Authors retain copyright and grant the journal right of first publication with the work. Reconstructive Review follows the Creative Commons Attribution-NonCommercial CC BY-NC. This license allows anyone to download works, build upon the material, and share them with others for non-commercial purposes as long as they credit the senior author, Reconstructive Review, and the Joint Implant Surgery \& Research Foundation (JISRF). 


\section{Introduction}

Total knee arthroplasty (TKA) is a successful treatment for advanced arthritis to remove pain and restore function. The longevity of the prosthetic components has been increasing, and the survival rate of the primary TKA was reported to be ranged from 89.5 to $95.3 \%$ at 10 years [1]. However, up to $20 \%$ of TKA patients were not satisfied with the outcome at 1 year post TKA [2], and unfortunately more than $35 \%$ of revision cases were performed within 2 years of primary TKA as early failures [3,4].

The failures in TKA have occurred for several reasons; aseptic loosening, polyethylene wear, instability, infection, arthrofibrosis, malalignment, malposition, deficient extensor mechanism, periprosthetic fracture, and patellar complications. Some of these factors could be minimized by design and material refinements of the components. However, many failures, involving malalignment, malposition and instability were also closely related to such surgical techniques as bone resection and soft tissue balancing [4$6]$.

For the bone resection, intramedullary femoral and extramedullary tibial alignment guides are most commonly applied. However, the limitations of the accuracy of these conventional methods have been of concern $[7,8]$. It has been estimated that $10 \%$ or more of TKAs have greater than 3 degrees error in lower-extremity mechanical axis and femoral and tibial component alignments, even when performed by experienced arthroplasty surgeons $[7,9]$.

To improve the accuracy of bone resections in TKA, a computer-assisted surgery (CAS) TKA was first performed in 1997 [10]. The device used for this procedure showed some promise for improvement of accuracy, and thereafter, related navigation technologies have continually improved the positioning of prosthetic components. However, most of these technologies have involved large and expensive equipment. Recently, a relatively low-cost, high reliability handheld device, the KneeAlign 2 (OrthAlign Inc., Aliso Viejo, CA), a compact accelerometer-based CAS, has been employed [15].

To obtain the appropriate soft tissue balancing, there are presently two schools of thought: 1) measured resection and 2) gap balancing. The first, the measured resection technique, employs femoral and tibial resections which are made independently based on anatomic bony references, i.e. transepicondylar axis (TEA), posterior condylar axis (PCA), and trochlear anteroposterior axis (Whiteside's line). Following that, soft tissue balancing is assessed with spacer blocks or trial prostheses. The second, the gap balancing technique, is based on the femoral component being positioned parallel to the resected proximal tibia with each collateral ligament equally tensioned by tensiometers or laminar spreaders [11]. These methodologies have been developed and modified through scientific studies. However, most of the procedures for soft tissue balancing still depend primarily upon the experience-based assessment of the surgeon's subjective "feel" as an "art form" $[12,13]$.

The eLibra (Zimmer Inc., Warsaw, IN) is an electronic knee balancing system, based on a relatively inexpensive handheld force sensor device to determine the rotation of the femoral component to obtain the appropriate balance by measuring the relative pressures within the medial and lateral compartments before the posterior femoral bone resection [12].

We hypothesized that a combination of the two relatively inexpensive handheld devices might achieve TKA with more precise bone resection and appropriate soft tissue balancing than the conventional procedures, i.e. without any technological devices, or with only one or the other of the two devices. The objective of this study, therefore, was to evaluate the accuracy of the surgical procedure by means of the combination of an accelerometer-based navigation and a soft tissue force sensor for TKA.

\section{Materials and Methods}

The inclusion criteria of this study were patients, who had been diagnosed with primary osteoarthritis and had been indicated for TKA. Five consecutive patients were enrolled as an experimental group. Under spinal anesthesia, all TKAs were carried out by a single surgeon (JF) in October 2014. Five consecutive TKAs, which had been performed by the same surgeon just before the experimental group, were enrolled as a control group. The exclusion criteria were patients with revision TKAs or secondary knee operation. One patient was excluded because of the past history of anterior cruciate ligament reconstruction surgery. All TKAs underwent midline skin incision with medial parapatellar arthrotomy.

The KneeAlign 2 system, which consists of a display console, a reference sensor, and femoral and tibial jigs, was applied for the distal femoral and proximal tibial resections (Fig. 1). For the distal femoral resection, the femoral jig and the distal femoral cutting block were seated at the midpoint of intracondylar notch on the anterior aspect of the posterior cruciate ligament attachment, which is similar to the insertion point for the conventional intramedullary femoral alignment guide system. The display console was attached to the femoral jig, and the reference sensor was attached to the cutting block. The display console and the reference sensor contain accelerometers and 


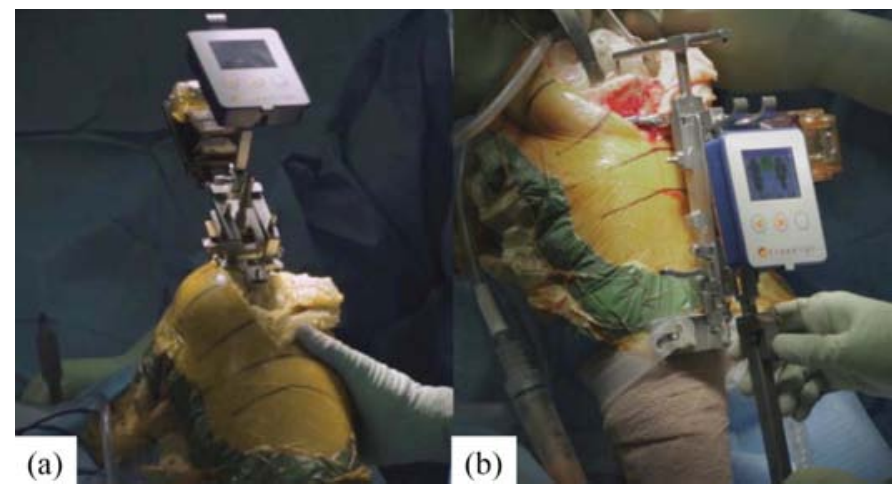

Fig. 1: The kneeAlign 2 navigation system consists of a display console, reference sensor and (a) femoral and (b) tibial jigs (Image provided by OrthAlign Inc., Aliso Viejo, California).

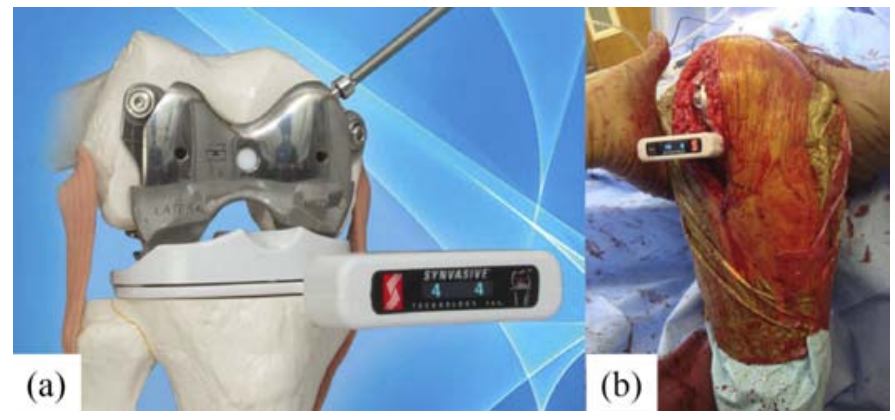

Fig. 2: (a) The eLibra electronic knee balancing system (Image provided by Zimmer, Warsaw, Indiana). (b) Insertion of the sensor in flexion, patella reduction, and leg drop off from the table for adjusting the soft tissue balancing.

communicate wirelessly with each other to determine the cutting block's alignment. After the registration of initial position of the cutting block, the hip center of rotation was determined by flexion/extension and internal/external rotation of the flexed hip. The cutting block was adjusted to 0 degrees in the coronal (varus/valgus) and sagittal (flexion/ extension) planes, perpendicular to the mechanical axis, and the distal femur was resected.

Patellae were resurfaced and replaced in all cases at this point before the proximal tibial osteotomy. The tibial jig was placed on the anterior aspect of the anterior cruciate ligament footprint to the proximal tibia, which is also similar to the conventional extramedullary tibial alignment guide system. The proximal tibial cutting block, the display console and the reference sensor were attached to the jig, and the components were fixed with a pin to the tibia. Following that, the tibial axis was determined by registering the anterior aspect of the anterior cruciate ligament footprint to the proximal tibia, and the medial and lateral malleoli. The cutting block was adjusted to 0 degrees in the coronal (varus/valgus) and 3 degrees in the sagittal (posterior slope) planes on the tibial axis, and the proximal tibia was resected.

For the control group, the distal femoral and the proxi- mal tibial resections were performed with intramedullary femoral and extramedullary tibial alignment guides. The intraoperative target of the distal femur was 5 degrees valgus from the anatomical axis in the coronal, and that of the proximal tibia was 0 degrees in the coronal and 3 degrees in the sagittal planes.

After the distal femoral, the proximal tibial, and the patellar resections were completed, the soft tissue balancing in knee extension was evaluated by the surgeon manually. Following that, the eLibra electronic knee balancing system, which comprises an electronic force sensor, the stainless steel eLibra femoral components and the tibial inserts, were applied (Fig. 2). The patella with a trial component was reduced, the thigh was lifted up, and the leg was dropped off from the table. The medial and lateral pressures were balanced by adjusting the sensor, and then the femoral external rotation angle was decided. The eLibra was then exchanged to the implant manufacturer's provided bone cutting block, and the final femoral resections were completed. The knee joint was taken through a full range of motion, and the intercompartmental load differential was examined with the placing of all trial components. The lateral retinaculum was released if the patella showed subluxation.

A fixed-bearing cruciate retaining 3DKnee system (DJO Global, Vista, CA) was implanted with bone cement. A pneumatic tourniquet was only inflated during curing of the cement, usually 12 to 15 minutes.

The standing anteroposterior hip to ankle, and the standing lateral knee to ankle radiographs were obtained 4 to 12 weeks after the surgery, from which the lower-extremity mechanical axis, femoral component varus/valgus alignment, tibial component varus/valgus alignment and posterior slope were digitally measured $[14,15]$. A picture archiving and communication system (PACS) imaging system (Philips Medical Systems, Sectra Imtec AB, Sweden) was adopted at our institute, and the unit of measurement was 1 degree.

For convention, a varus alignment between the measurement angle and 180 degrees for the mechanical axis and 90 degrees for components was given a positive numerical value; a valgus alignment was given a negative numerical value. Tibial slope was designated negative for an anterior slope and positive for a posterior slope. Target intraoperative alignment of the distal femoral coronal (varus/ valgus) and the sagittal (flexion/extension) planes were 0 degrees, and the proximal tibial coronal (varus/valgus) and the sagittal (posterior/anterior slope) planes were 0 and 3 degrees, respectively. Any values greater than 3 degrees from the intraoperative goal were classified as outliers. 


\section{Statistical Analysis}

Descriptive statistics were used to describe the difference in the mechanical, femoral and tibial axes, conducted by the paired samples t-tests between the experimental and the control groups. All statistical analyses were performed with SPSS version 19.0 software (SPSS Inc., Chicago, IL) and significance level was set at $\mathrm{p}<0.05$.

\section{Results}

Values are expressed as mean \pm standard deviation.

In the experimental group, there were 1 female and 3 males. One male patient underwent simultaneous bilateral TKAs and these cases were examined separately. Average age was $75 \pm 2.6$ (ranging from 73 to 79 ), and BMI was $34.7 \pm 7.9$ (26.6 to 43.1 ).

Operation time was $96.8 \pm 11$ (85 to 111) minutes from the skin incision to the skin suture. Pneumatic tourniquet time was $13.6 \pm 1.3$ (12 to 15 ) minutes.

The femoral CAS process from the preparation of the femoral jigs to the distal femoral resection was $8.2 \pm 1.6$ (6 to 10) minutes. The tibial CAS process from the preparation of the tibial jigs to the proximal tibial resection was $12.4 \pm 3.4$ (9 to 17) minutes. In one case, the proximal tibia was additionally recut because of inadequate posterior slope after the first cut under the setting of 3 degrees with KneeAlign 2, and thus, it took 17 minutes in total.

On the other hand, the force sensor process from the preparation of the sensor to the exchange to the manufacturer's cutting block was $3.6 \pm 0.5$ (3 to 4 ) minutes. The external rotation angle of the femur was $5.2 \pm 1.3$ ( 3 to 6 ) degrees.

The radiographic findings showed that the lower-ex-

(a)

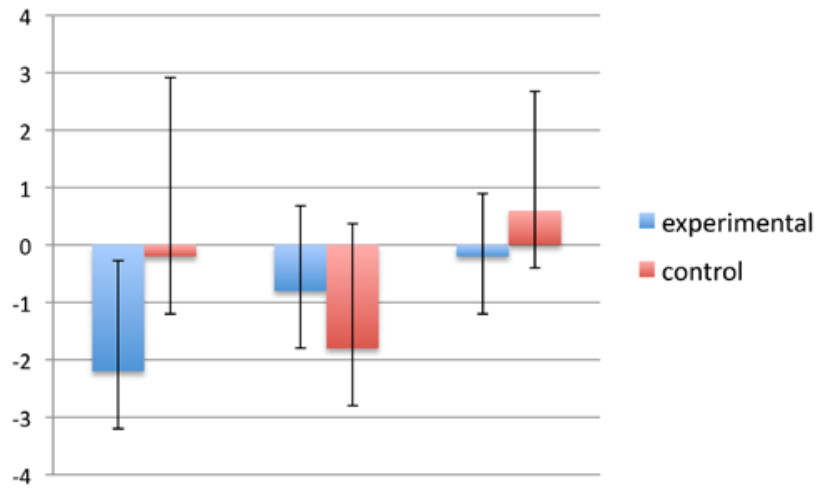

(1) tremity mechanical axis was 2.2 degrees valgus alignment (-4 to +1$)$, femoral component was 0.8 degrees valgus alignment $(-3$ to +1$)$, tibial component was 0.2 degrees valgus alignment $(-1$ to +1$)$, and posterior slope was 2.6 degrees ( 1 to 5) (Fig. 3). The mechanical alignment in 1 case was 4 degrees valgus, which was diagnosed as an outlier. Otherwise, all other parameters were less than 3 degrees, which were within the normal range.

In the control group, there were 4 males, and 1 patient underwent simultaneous bilateral TKAs, and these cases were examined separately. Their average age was $63 \pm$ 14.8 (51 to 83), and BMI was $26.6 \pm 2.5$ (22.2 to 28.7).

The radiographic findings showed that the mechanical axis was 0.2 degrees valgus alignment $(-4$ to +3$)$, femoral component was 1.8 degrees valgus alignment $(-4$ to +1$)$, tibial component was 0.6 degrees varus alignment ( -2 to +3 ), and posterior slope was 6.8 degrees (5 to 9) (Fig. 3). As outliers, one patient showed 4 degrees valgus of mechanical axis, 4 degrees valgus of femoral component, and 9 degrees of posterior slope, while another patient showed 8 degrees of posterior slope. The posterior slope of the tibia was significantly improved in the experimental group compared to the control group ( $\mathrm{p}=0.004)$. However, the mechanical, femoral and tibial axes were not statistically different between the experimental and control groups.

There were no lateral retinacular releases performed in either group. Moreover, there were no significant post TKA complications, infections nor venous thromboembolism suffered in any cases.

\section{Discussion}

To improve the accuracy of TKA, the combination of an accelerometer-based navigation and a soft tissue force sen-

(b)

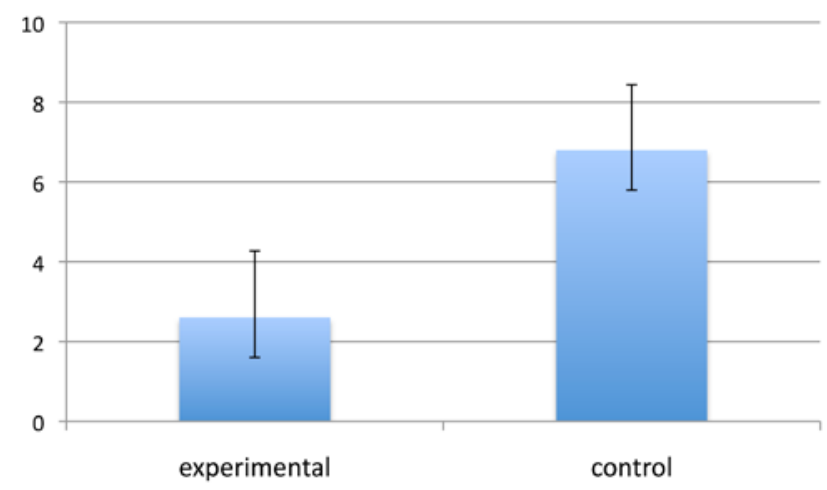

Fig. 3: Comparison between the experimental and the control groups. (a)-(1) mechanical axis, (2) femoral alignment, (3) tibial alignment. (b) posterior slope of the tibia. The posterior tibial slope was significantly improved compared to the control group. 
sor were applied to the procedure. Only one radiographic outlier occurred in a single patient in the CAS experimental group. Moreover, soft tissue balances were correctly adjusted without lateral release by use of the force sensor.

Malalignment TKA has been reported to be implicated in polyethylene wear and aseptic implant loosening [1619]. The external rotation of the femoral component is determined by appropriate soft tissue balancing combined with accurate bone resection. On the other hand, malposition with inappropriate soft tissue balancing results in patellar maltracking, femoral component lift off, anterior knee pain, and knee instability, especially in flexion [11]. Therefore, it is imperative to achieve precise bone alignment and appropriate soft tissue balancing for optimal outcomes in TKA.

Many articles have demonstrated the increased accuracy of bony alignment by CAS compared to the conventional maneuvers $[7,8]$. Recently, the improvement of clinical function with the accurate mechanical alignment by CAS was published [20]. However, CAS involves some detriments; increased capital cost, longer procedure times, preoperative additional image examinations, intraoperative difficulties of handling bulky devices or sensitive instruments, steep learning curves, and potential tracer pin site complications $[5,21]$.

The accelerometer-based navigation has been adopted, firstly because its accuracy and efficacy have already been confirmed $[15,24]$. Secondly, these devices are easy to be operated by most orthopedic surgeons with some TKA experience, because these devices are handheld and the maneuvers of bone resections with them are similar to the conventional techniques with intramedullary femoral and extramedullary tibial guides It has been reported that the time for pinning the tibial cutting block was 3.4 minutes and for completion of tibial resection was 4.6 minutes while using this device, and thus it was considered not to be longer than the conventional instrumentation [24]. In our experimental group, it took 8.2 minutes for femoral and 12.4 minutes for tibial resections, including setting up devises, adjusting the cutting blocks, and cutting the bones. Thus we also deemed these processes to be similar to the conventional methods. Thirdly, based on the accelerometer, the anatomical references can be registered intraoperatively without screws. Moreover, the femoral guide is simply attached to the femoral surface, with no need for intramedullary insertion. Thus, preoperative image preparations and insertions of screws are not required, eliminating the causes of fatty emboli production. Fourthly, this system is compatible with most manufacturers' prostheses.

Recently, it has been tried to involve the process to adjust soft tissue balancing in CAS $[13,25]$, however, most of the CAS, including KneeAlign 2, have not been designed for soft tissue balancing. Moreover, it is difficult to determine the proper alignment with mediolateral stability in flexion for a hypoplastic lateral condyle with a severe valgus knee deformity [26]. Therefore, the appropriate soft tissue balancing with proper rotation of the femur is a prerequisite for successful TKAs, in addition to the precise bone resections.

For soft tissue balancing, the eLibra knee balancing device has been adopted, firstly because of its efficacy, reproducibility and reduction in need for lateral release [27]. The lateral release is a simple procedure to improve patellar tracking, however, it involves patellar complications; e.g. patellar hypovascularity, fracture and component loosening. Because the flexion gap was adjusted before the posterior femoral resection, the incidence of lateral release was reported to be reduced from $12 \%$ to $3 \%$ with this device [12]. In this study, the patellar stability was achieved, without the lateral release throughout a full range of knee motion for all cases in both groups. Secondly, the eLibra device is intuitive and handheld similar to the conventional spacer blocks. Thirdly, it has been reported to take only an additional 3 minutes to accurately achieve the soft tissue balancing with this device [27]. The technique is easier to learn than the gap balancing technique. It took $3.6 \mathrm{~min}$ utes in the experimental group, and it does not affect the total surgical time. Fourthly, this system is compatible with most of the manufacturers' prostheses.

The cost is another inevitable argument for hospitals. Most of the conventional CAS devices are expensive, and can be used only at hospitals with high volume of TKAs. However, most hospitals are not that high in volume. Moreover, most TKA surgeons are not arthroplasty specialists only performing high numbers of arthroplasties. Because both of these systems are inexpensive disposable single-use devices, they are available to apply even in hospitals with low numbers of cases.

This study includes several limitations. This was a preliminary study of cases performed by a single surgeon, with a small number of patients and a short-term followup. Thus, we were unable to determine if the accurate TKAs were beneficial for long-term prosthetic survival or the clinical outcomes. However, malalignment, malposition, and instability are definitely factors related to early failure of TKA. Therefore, this data showed the possibility of improving the outcome of TKAs with precise bone resection and appropriate soft tissue balancing.

Traditional CAS systems rely on anatomic registration points to reconstruct the knee. Incorrect selection of reference landmarks results in component malposition and balancing issues. In this study, an additional posterior tibia 
cut was performed for one case in the experimental group, because of an inadequate posterior slope of the tibia. It was considered that the register point on the tibia surface was selected more posteriorly than expected. To select inadequate reference landmarks is a pitfall for TKA, and therefore, surgeons always need sufficient anatomical and surgical knowledge and must verify the alignment using conventional guides step by step, even under CAS TKA.

\section{Conclusions}

The combination of an accelerometer-based navigation and a soft tissue force sensor was applied for TKA. Using these technologies, precise bone alignment and appropriate soft tissue balancing can be obtained.

\section{Disclosure}

The authors declare that there are no conflict of interest regarding the publication of this paper. For full disclosures refer to last page of this journal.

\section{References}

1. Gothesen O, Espehaug B, Havelin L, Petursson G, Lygre S, Ellison P, Hallan G, Furnes $\mathrm{O}$. Survival rates and causes of revision in cemented primary total knee replacement: a report from the Norwegian Arthroplasty Register 1994-2009. Bone Joint J. 2013 May;95-B(5):636-42.

2. Scott CE, Howie CR, MacDonald D, Biant LC. Predicting dissatisfaction following total knee replacement: a prospective study of 1217 patients. J Bone Joint Surg Br. 2010 Sep;92(9):1253-8.

3. Lombardi AV Jr, Berend KR, Adams JB. Why knee replacements fail in 2013 patient, surgeon, or implant? Bone Joint J. 2014 Nov;96-B(11 Supple A):101-4.

4. Sharkey PF, Lichstein PM, Shen C, Tokarski AT, Parvizi J. Why are total knee arthroplasties failing today--has anything changed after 10 years? J Arthroplasty. 2014 Sep;29(9):1774-8.

5. Clayton AW, Cherian JJ, Banerjee S, Kapadia BH, Jauregui JJ, Harwin SF, Mon MA. Does the use of navigation in total knee arthroplasty affect outcomes? J Knee Surg. 2014 Jun;27(3):171-5.

6. Moreland JR. Mechanisms of failure in total knee arthroplasty. Clin Orthop Relat Res. 1988 Jan;(226):49-64.

7. Anderson KC, Buehler KC, Markel DC. Computer assisted navigation in total knee arthroplasty: comparison with conventional methods. J Arthroplasty 2005;20 (7, Suppl 3):132-138

8. Blakeney WG, Khan RJ, Wall SJ. Computer-assisted techniques versus conventional guides for component alignment in total knee arthroplasty: a randomized controlled trial. J Bone Joint Surg Am. 2011 Aug 3;93(15):1377-84.
9. Mahaluxmivala J, Bankes MJ, Nicolai P, Aldam CH, Allen PW. The effect of surgeon experience on component positioning in 673 Press Fit Condylar posterior cruciate-sacrificing total knee arthroplasties. J Arthroplasty. 2001 Aug;16(5):63540 .

10. Krackow KA, Bayers-Thering M, Phillips MJ, Bayers-Thering M, Mihalko WM. A new technique for determining proper mechanical axis alignment during total knee arthroplasty: progress toward computer-assisted TKA. Orthopedics 1999;22(7):698-702

11. Daines BK, Dennis DA. Gap balancing vs. measured resection technique in total knee arthroplasty. Clin Orthop Surg. 2014 Mar;6(1):1-8.

12. Fetto JF, Hadley S, Leffers KJ, Leslie CJ, Schwarzkopf R. Electronic measurement of soft-tissue balancing reduces lateral releases in total knee arthroplasty. Bull NYU Hosp Jt Dis. 2011;69(4):285-8.

13. Joseph J, Simpson PM, Whitehouse SL, English HW, Donnelly WJ. The use of navigation to achieve soft tissue balance in total knee arthroplasty - a randomised clinical study. Knee. 2013 Dec;20(6):401-6.

14. Nam D, Nawabi DH, Cross MB, Heyse TJ, Mayman DJ. Accelerometer-based computer navigation for performing the distal femoral resection in total knee arthroplasty. J Arthroplasty. 2012 Oct;27(9):1717-22.

15. Nam D, Cody EA, Nguyen JT, Figgie MP, Mayman DJ. Extramedullary guides versus portable, accelerometer-based navigation for tibial alignment in total knee arthroplasty: a randomized, controlled trial: winner of the 2013 HAP PAUL award. J Arthroplasty. 2014 Feb;29(2):288-94.

16. Jeffery RS, Morris RW, Denham RA. Coronal alignment after total knee replacement. J Bone Joint Surg Br. 1991 Sep;73(5):709-14.

17. Ritter MA, Faris PM, Keating EM, Meding JB. Postoperative alignment of total knee replacement. Its effect on survival. Clin Orthop Relat Res. 1994 Feb;(299):153-6.

18. Wasielewski RC, Galante JO, Leighty RM, Natarajan RN, Rosenberg AG. Wear patterns on retrieved polyethylene tibial inserts and their relationship to technical considerations during total knee arthroplasty. Clin Orthop Relat Res. 1994 Feb;(299):31-43.

19. Berend ME, Ritter MA, Meding JB, Faris PM, Keating EM, Redelman R, Faris GW, Davis KE. Tibial component failure mechanisms in total knee arthroplasty. Clin Orthop Relat Res. 2004 Nov;(428):26-34

20. Rebal BA, Babatunde OM, Lee JH, Geller JA, Patrick DA Jr, Macaulay W. Imageless computer navigation in total knee arthroplasty provides superior short term functional outcomes: a meta-analysis. J Arthroplasty. 2014 May;29(5):938-44.

21. Ossendorf C, Fuchs B, Koch P. Femoral stress fracture after computer navigated total knee arthroplasty. Knee 2006;13(5): 397-399

22. Kim YH, Kim JS, Choi Y, Kwon OR. Computer-assisted surgical navigation does not improve the alignment and orientation of the components in total knee arthroplasty. J Bone Joint Surg Am. 2009 Jan;91(1):14-9.

23. Johnson DR, Dennis DA, Kindsfater KA, Kim RH. Evaluation of total knee arthroplasty performed with and without computer navigation: a bilateral total knee arthroplasty study. J Arthroplasty. 2013 Mar;28(3):455-8.

24. Bugbee WD, Kermanshahi AY, Munro MM, McCauley JC, Copp SN. Accuracy of a hand-held surgical navigation system for tibial resection in total knee arthroplasty. Knee. 2014 Dec;21(6):1225-8

25. Saragaglia D, Chaussard C, Rubens-Duval B. Navigation as a predictor of soft tissue release during 90 cases of computer-assisted total knee arthroplasty. Orthopedics. 2006 Oct;29(10 Suppl):S137-8.

26. Stephens BF, Hakki S, Saleh KJ, Mihalko WM. Clinical alignment variations in total knee arthroplasty with different navigation methods. Knee. 2014 Oct;21(5):971-4.

27. Camarata DA. Soft tissue balance in total knee arthroplasty with a force sensor Orthop Clin North Am. 2014 Apr;45(2):175-84. 